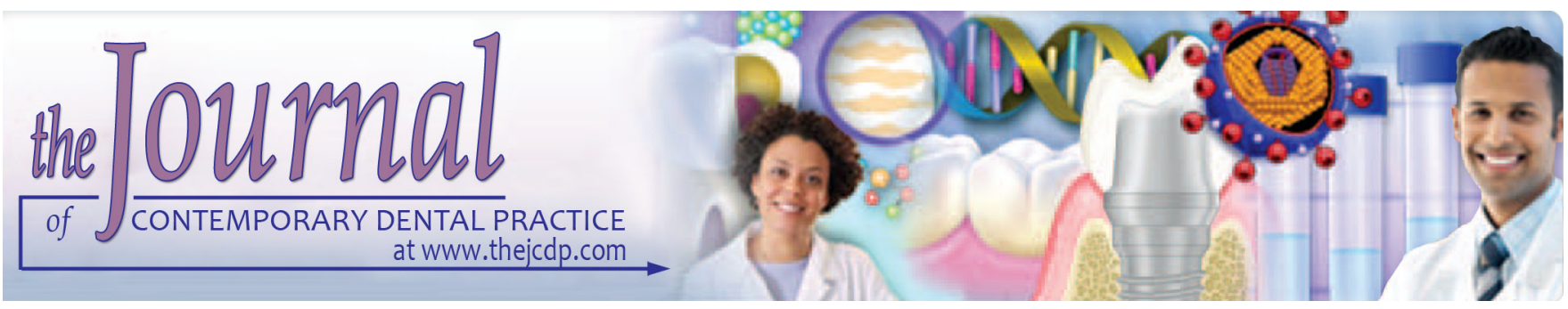

\title{
Nickel-Titanium Single-file System in Endodontics
}

\section{Alberto Dagna}

\section{ABSTRACT}

Aim: This work describes clinical cases treated with a innovative single-use and single-file nickel-titanium (NiTi) system used in continuous rotation.

Background: Nickel-titanium files are commonly used for root canal treatment but they tend to break because of bending stresses and torsional stresses. Today new instruments used only for one treatment have been introduced. They help the clinician to make the root canal shaping easier and safer because they do not require sterilization and after use have to be discarded. A new sterile instrument is used for each treatment in order to reduce the possibility of fracture inside the canal. The new One Shape NiTi single-file instrument belongs to this group.

Case description: One Shape is used for complete shaping of root canal after an adequate preflaring. Its protocol is simple and some clinical cases are presented. It is helpful for easy cases and reliable for difficult canals.

Conclusion: After 2 years of clinical practice, One Shape seems to be helpful for the treatment of most of the root canals, with low risk of separation. After each treatment, the instrument is discarded and not sterilized in autoclave or re-used.

Clinical significance: This single-use file simplifies the endodontic therapy, because only one instrument is required for canal shaping of many cases. The respect of clinical protocol guarantees predictable good results.

Keywords: Nickel-titanium instruments, Root canal shaping, Single-file technique, Single-use file.

How to cite this article: Dagna A. Nickel-Titanium Single-file System in Endodontics. J Contemp Dent Pract 2015;16(10): 834-839.

Source of support: Nil

Conflict of interest: None

Department of Clinical-Surgical, Diagnostic and Pediatric Sciences, Section of Dentistry; Department of Endodontic University of Pavia, Italy

Corresponding Author: Alberto Dagna, Assistant Professor Department of Clinical-Surgical, Diagnostic and Pediatric Sciences, Section of Dentistry; Department of Endodontic University of Pavia, Italy, Phone: +39 0131 317071, e-mail: alberto.dagna@unipv.it

\section{BACKGROUND}

Root canal shaping is one of the most important steps in canal treatment and the nickel-titanium (NiTi) instruments have become a standard tool in modern endodontics. ${ }^{1}$ The superelasticity of NiTi instruments permits to gene rate a constant tapered root $\mathrm{canal}^{2}$ but in clinical practice they are always exposed to the risk of fracture. ${ }^{3}$ Separation may be caused by cyclic fatigue because of bending and by shear stresses exceeding the resistance of the alloy because of torque. ${ }^{4,5}$ These stresses become higher when an unnecessary pressure is applied on the handpiece by the operator, ${ }^{6}$ when the interface area between the dentin walls and the edges of the instrument widens or when the canal diameter is smaller than the section of the file: ${ }^{7}$ this causes a phenomenon called 'taper lock' ${ }^{8}$ This probability may be reduced by enlarging the coronal third ${ }^{9}$ and by creating an adequate glide path before using each NiTi rotary instrumentation. ${ }^{10,11}$

The new single-file technique has been recently introduced to simplify instrumentation protocols, to reduces stresses and to avoid the risk of cross-contamination. ${ }^{11}$ However, root canal shaping with only one file submit the instruments to a great deal of torsional and flexural stresses. ${ }^{12}$ Every file is not used for further canals and not requires thermal sterilization in autoclave, so it will be not exposed to other stresses.

The One Shape (Micro Mega, Besancon, France) is made of a conventional austenite 55-NiTi alloy but it's a single-use and a single-file system developed for a full clockwise rotation. It consists of only one instrument, with a tip size of 25 and 0.06 constant taper. The main characteristic is the asymmetrical cross-sectional design of the working portion. One Shape has three cutting edges in the tip region but in the middle the cross-sectional design progressively changes from a three-cutting-edges design to two cutting edges: at the shank it has two cutting edges with a S-shaped cross section. Moreover, the One Shape is characterized from variable pitch length of the 
working part. It is developed for using in continuous clockwise rotation with rotational speed at 350 to $450 \mathrm{rpm}$ and torque setting at $4 \mathrm{Ncm}$. Its protocol requires an adequate preflaring before the root canal shaping: after that, it is introduced into the root canal with a slow and passive brushing motion: it is necessary an upward circumferential filing movement, when possible, until the working length is reached. Frequent withdrawal from the root canal and cleansing of the blades is very important. Copious irrigation of root canal system is necessary to remove dentinal debris.

\section{CASE DESCRIPTION}

Few instruments are needed to complete the root canal shaping of a simple case: some stainless steel hand files for canal scouting and working length determination and then the One Shape. But depending on the anatomy and the complexity of each case, the numbers of files may increase. Many instruments are needed when it is difficult to reach the apex or when obstacles are present along the root canal (Figs 1A to C): the apical constriction should be bypassed with hand files or with NiTi instruments for preflaring, and then One Shape is free to shape the entire root canal. Alternatively, when the root canal is easy and the apical diameter is increased more instruments are needed to complete the cleansing and shaping of the apical third (Figs 2A and B). If the protocol is respected, the instrument is capable to shape easily root canal with serious curvature (Figs 2C and D). Some cases require other NiTi instruments with reduced taper to reach the
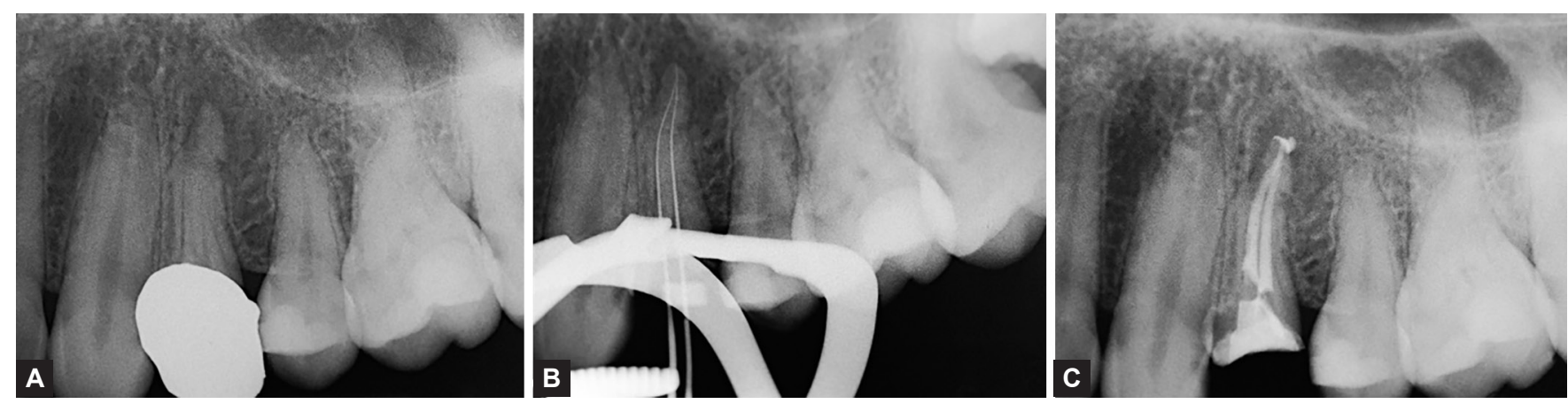

Figs $1 \mathrm{~A}$ to $\mathrm{C}$ : First maxillary premolar: (A) Preoperative $\mathrm{Rx},(\mathrm{B})$ intraoperative $\mathrm{Rx}$ for $\mathrm{WL}$ control and $(\mathrm{C})$ postoperative $\mathrm{Rx}$ many hand files were necessary to reach the apex, a G1G-file was used for preflaring, The One Shape was used for canal shaping and a \#25 K-file was used for apical finishing

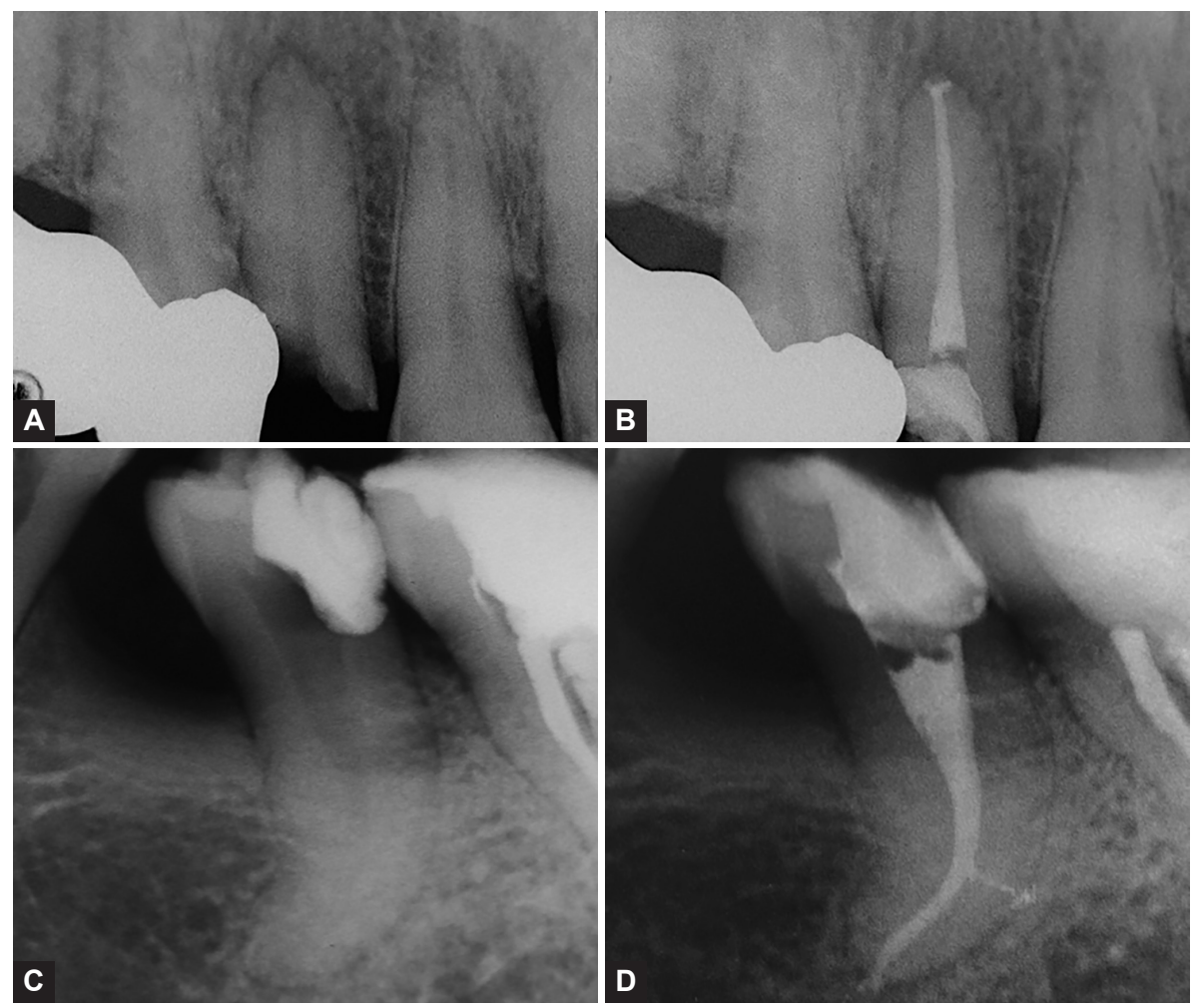

Figs 2A to D: (A) Preoperative Rx of maxillary lateral incisor, (B) postoperative Rx: some stainless steel K-files and one Micro-Mega Revo-S AS35 NiTi file with increased diameter were necessary for apical finishing after the One Shape was used, (C) preoperative Rx of second maxillary premolar with curvature in the apical third and (D) postoperative Rx after shaping with One Shape 3 
working length (Figs 3A to C): a lower premolar with double curvature of root requires a meticulous preflaring with precurved stainless steel hand files and apposite NiTi rotary files with low apical diameter and reduced taper, than a $4 \%$ taper NiTi is necessary to shape the root canal before the use of One Shape.

When is possible, two teeth can be treated with the same instrument in a single visit (Figs 3D to F).
One Shape is useful also for endodontic retreatment. Figures $4 \mathrm{~A}$ to I show step-by-step the retreatment of a first mandibular molar with incomplete obturation and periradicular lesion. After removal of old crown restoration and resin composite build-up of mesial wall, the access cavity was prepared and refined with an ultrasonic device, so all orifices were opened. All canals were probed with a stainless steel \#10 K-file until the apical third and their
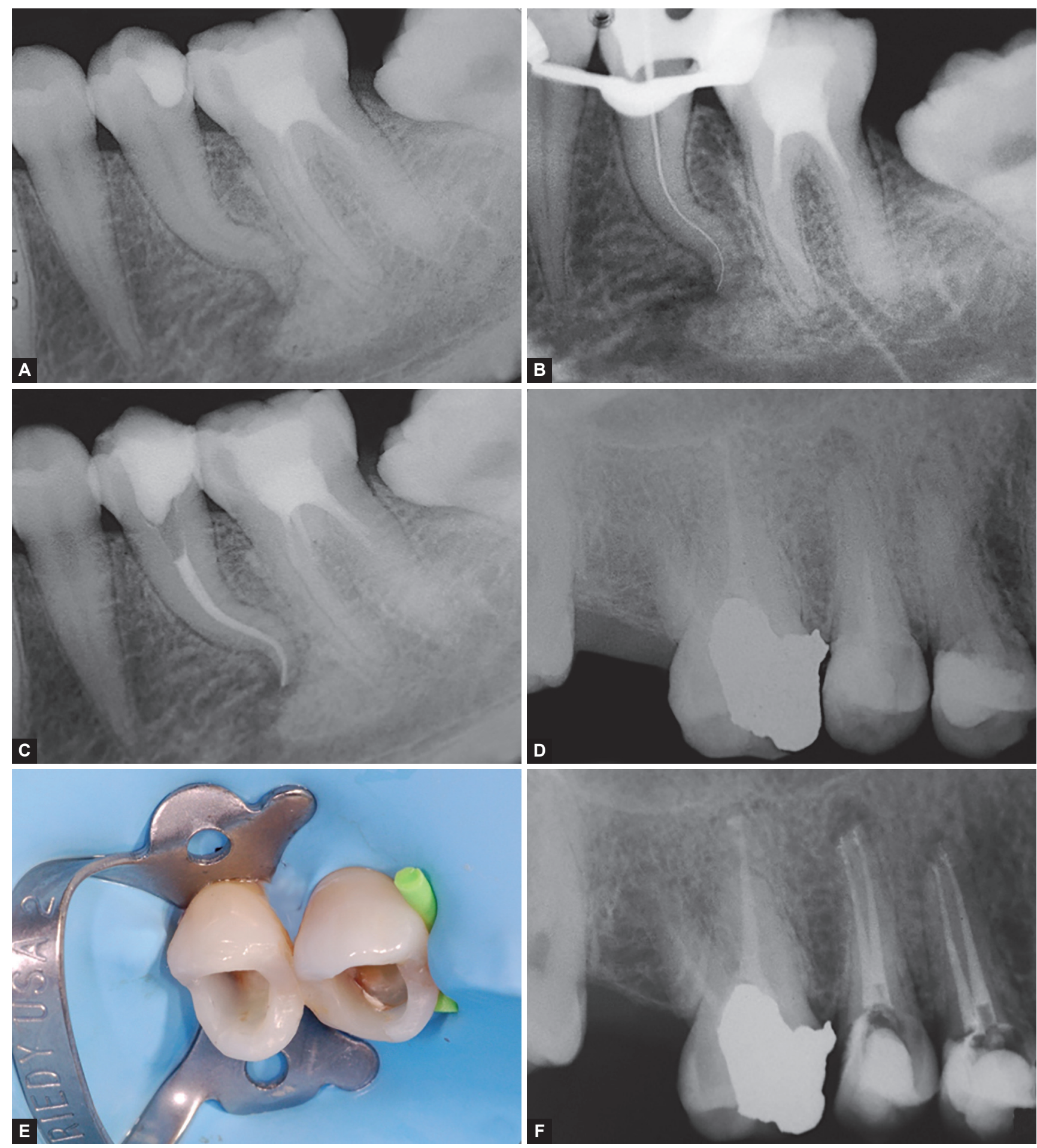

Figs 3A to F: (A) Preoperative Rx of second mandibular premolar with double root curvature, (B) intraoperative Rx with stainless steel K-file at WL, (C) postoperative Rx: three K-files to reach the apex, an Endoflare for orifice widening, a G1G-file for preflaring, a MicroMega Revo-S SC2 with a taper of 0.04 to bypass the second curve and the One Shape for canal shaping, (D) preoperative Rx of two maxillary premolars, $(E)$ intraoperative view: isolation with rubber dam of both premolars for single-visit treatment and $(F)$ postoperative $R x$ 

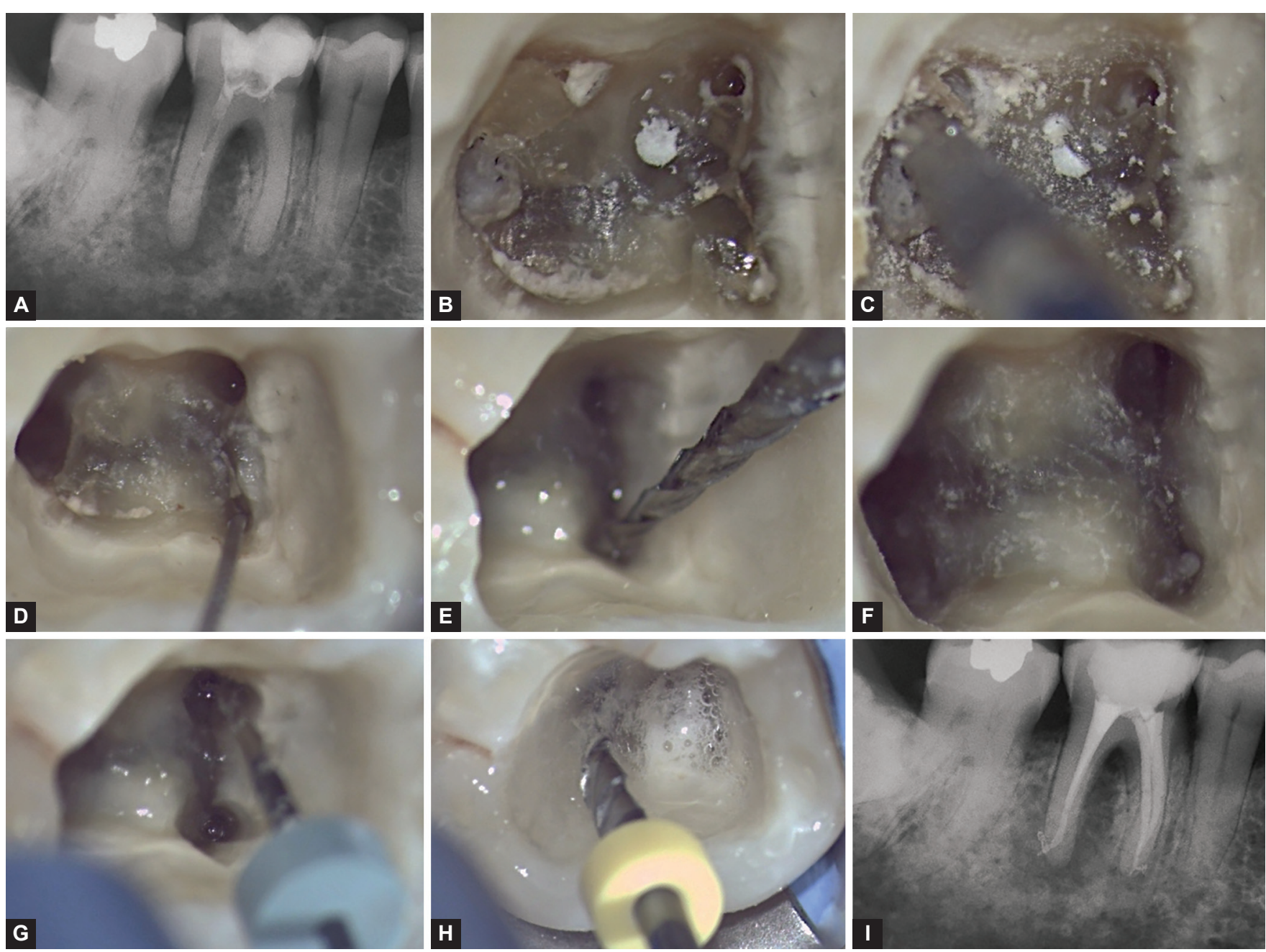

Figs 4A to I: Retreatment of mandibular first molar: (A) preoperative $\mathrm{Rx},(\mathrm{B})$ access cavity after buildup of mesial wall, $(\mathrm{C})$ access cavity finishing with ultrasonic tip, (D) scouting of canal orifices with a stainless steel no. $10 \mathrm{~K}$-file, (E) orifices widening with Endoflare, $(F)$ aspect of orifices after shaping with Endoflare, $(\mathrm{G})$ preflaring with $\mathrm{G} 1 \mathrm{G}$-file, $(\mathrm{H})$ One Shape at work and (I) postoperative Rx

orifices were prepared with the Endoflare (Micro-Mega, Besancon, France). Endoflare is a short NiTi file (tip size 25 and 0.12 constant taper) developed for orifice widening and coronal third shaping. Mechanical preflaring with G1G-file NiTi rotary instrument (Micro Mega, Besancon, France) was performed. G1G-file has a tip size of 12 and

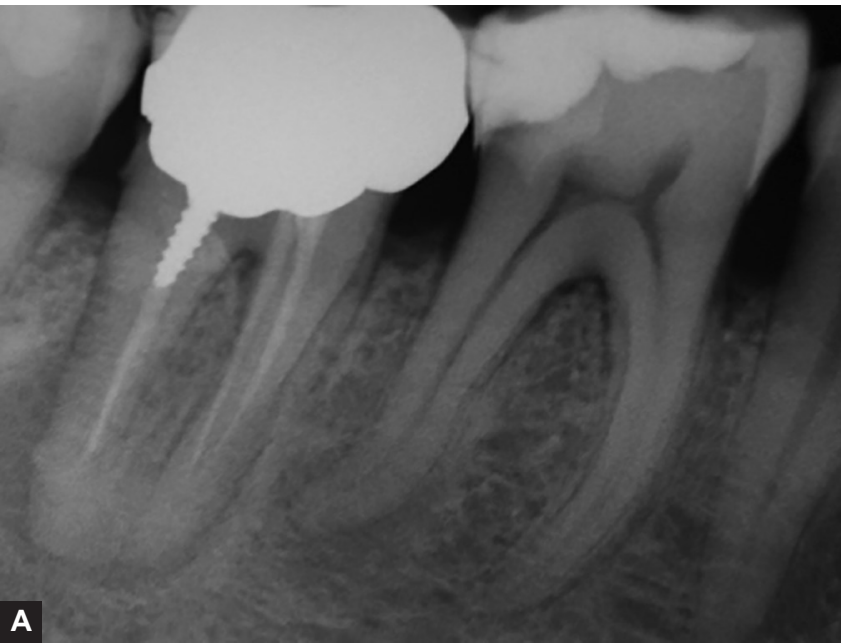

a constant taper of 0.03 with asymmetrical cross section. When the working length has been determined, all canals were shaped with One Shape according to manufacturer's protocol. Figures $5 \mathrm{~A}$ and $\mathrm{B}$ show the retreatment of a lower second molar made with One Shape after removal of prosthetic crown and renegotiation

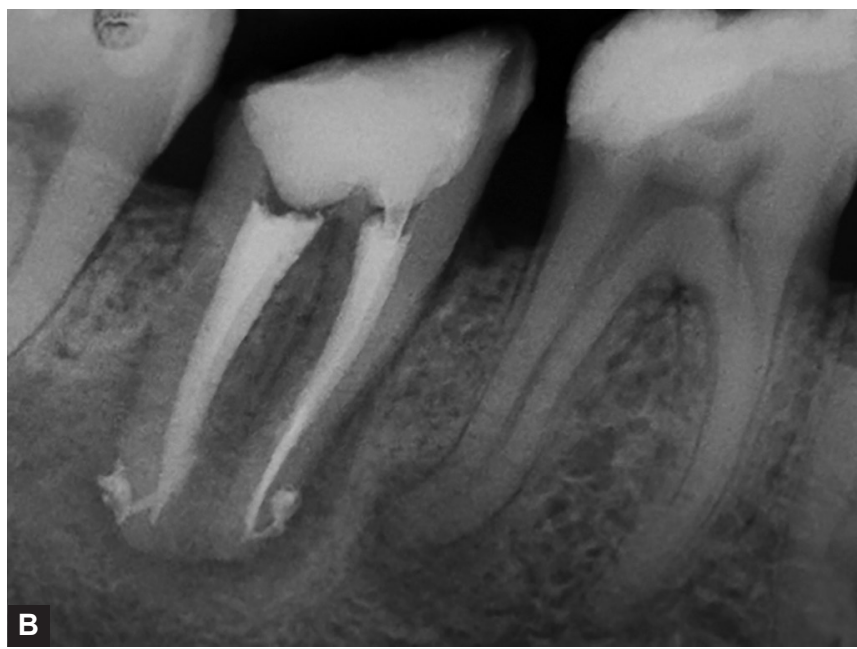

Figs 5A and B: Retreatment of mandibular second molar: (A) Preoperative Rx and (B) postoperative $\mathrm{Rx}$ 
of root canals with ultrasonic tips and stainless steel hand K-files.

One Shape is helpful in difficult cases. Figures $6 \mathrm{~A}$ to $\mathrm{F}$ show first mandibular molar with complex anatomy and tight canals. After caries removal and after restoration of mesial and distal walls with composite resin, the access cavity was prepared and finished using ultrasonic tips. Two mesial orifices and two distal orifices were detected and canal patency was checked with a stainless steel \#10
K-file. Endoflare enlarged the coronal third of each root canal and G1G-file created the glide path to the apex. Three G-files were discarded because of their deformation after removal from the root canals. After the glide path was established and the working length was determined, One Shape was carefully introduced in each canal using an in and out motion with no pressure and frequently withdrawn and cleansed alternating copious irrigation with $17 \%$ EDTA and $5 \% \mathrm{NaOCl}$. At each removal of
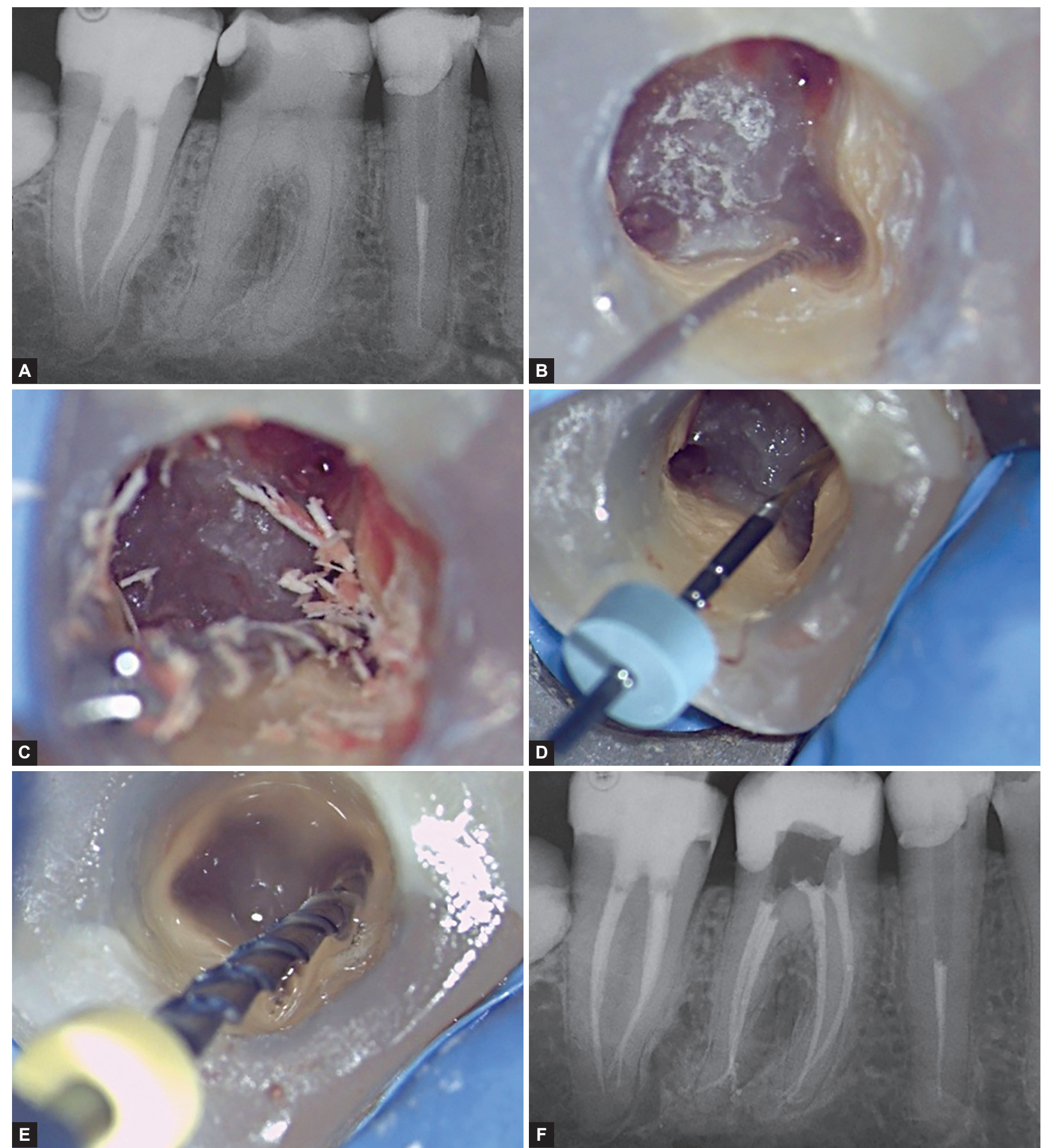

Figs 6A to F: Maxillary second molar with narrow canals: (A) Preoperative Rx, (B) canal scouting of orifices with \#10 K-file, (C) orifices widening with Endoflare, (D) preflaring with G1G-file, (E) One Shape at work and $(F)$ postoperative Rx 
One Shape root canal patency was checked with a \#10 K-file. At the end of the action of One Shape, the apical gauging was checked using a \#25 K-file and obturation was performed.

\section{DISCUSSION}

One Shape has been introduced to prepare majority of root canals with only one file. It is a single-use instrument, discarded after the treatment and not sterilized in autoclave for further treatments. This allows reducing the risks related to the stresses that conduce to the breakage of the file. ${ }^{3,4}$

The reciprocating motion is based on a cutting action in counterclockwise direction alternated to the release of the file in clockwise direction with a smaller degree of rotation: in this way, the cutting angle is greater than the angle of contra-rotation and the file continuously progresses to apical direction. ${ }^{11,12}$ Reciprocating movement was presented to extend the lifespan and the fatigue resistance of NiTi instruments ${ }^{12}$ and was presented as respectful of root canal anatomy with satisfactory shaping ability. ${ }^{11}$ Today some limitations regarding reciprocating systems were found. Burklein and Schafer ${ }^{13}$ verified that reciprocating files produce significantly more debris than traditional rotary systems during root canal shaping and their motion promote apical extrusion of debris. The extrusion of dentin chips, pulp tissue, microorga nisms and/or irrigating solutions may potentially cause postoperative complications. ${ }^{14}$ Other studies showed that reciprocating instruments produce a lot of smear layer along the dentinal walls, much more than conventional rotating instruments, so the cleansing and the disinfection of root canal system could be not effective. ${ }^{14,15}$

One Shape is the newest single-file instrument, based on traditional full clockwise rotation. It belongs to the group of single-use files, but it can be used with a conventional endodontic engine. So, it combines the advantages of single-use instruments to the experience and the benefits of continuous rotating motion. Its protocol is very easy and its use safe. After each treatment, the instrument is discarded and not re-used. Each treatment is performed with a new and not stressed instrument, so the risk of breakage or separation is very low.

\section{CONCLUSION}

Single-use NiTi instruments are a good tool for daily endodontics. One Shape simplify root canal shaping procedures thanks to its easy operative protocol. It is helpful for many clinical cases with good results. It is safe, because the risk of breakage is low. It is convenient and suitable for each traditional endodontic engine.

\section{CLINICAL SIGNIFICANCE}

The single-file technique simplifies the endodontic therapy, because only one instrument is required for canal shaping of many cases. A single-use instrument developed for continuous rotation does not require a dedicated engine unlike reciprocating instruments. The respect of clinical protocol permits the safe use of the file and guarantees predictable good results of endodontic treatment.

\section{REFERENCES}

1. Torabinejad M, Walton RE. Endodontics: principles and practice. 4th ed. St Louis, Missouri: Saunders Elsevier; 2009.

2. Peters OA. Current challenges and concepts in the preparation of root canal systems: a review. J Endod 2004;30:559-567.

3. Sattapan B, Nervo GJ, Palamara JE, Messer HH. Defects in rotary nickel-titanium files after clinical use. J Endod 2000;26:161-165.

4. Berutti E, Chiandussi G, Gaviglio I, Ibba A. Comparative analysis of torsional and bending stresses in two mathematical models of nickel-titanium rotary instruments: proTaper versus ProFile. J Endod 2003;29:15-19.

5. Parashos $\mathrm{P}$, Messer HH. Rotary NiTi instrument fracture and its consequences. J Endod 2006;32:1031-1043.

6. Kobayashi C, Yoshioka T, Suda H. A new engine-driven canal preparation system with electronic canal measuring capability. J Endod 1997;23:751-754.

7. Peters OA, Peters CI, Schonenberger K, Barbakow F. ProTaper rotary root canal preparation: assessment of torque and force in relation to canal anatomy. Int Endod J 2003;36:93-99.

8. Yared GM, Bou Dagher FE, Machtou P. Influence of rotational speed, torque and operator's proficiency on ProFile failure. Int Endod J 2001;34:47-53.

9. Roland DD, Andelin WE, Browning DF, Hsu GH, Torabinejad M. The effect of preflaring on the rates of separation for 0.04 taper nickel-titanium rotary instruments. J Endod 2002;28:543-545.

10. Berutti E, Negro AR, Lendini M, Pasqualini D. Influence of manual preflaring and torque on the failure rate of ProTaper rotary instruments. J Endod 2004;30:228-230.

11. Berutti E, Chiandussi G, Paolino DS, Scotti N, Cantatore G, Castellucci A, Pasqualini D. Canal shaping with WaveOne reciprocating files and ProTaper system: a comparative study. J Endod 2012;38:505-509.

12. Kim HC, Kwak SW, Cheung GS, Ko DH, Chung SM, Lee W. Cyclic fatigue and torsional resistance of two new nickeltitanium instruments used in reciprocation motion: Reciproc versus WaveOne. J Endod 2012;38:541-544.

13. Burklein S, Schafer E. Apically extruded debris with reciprocating single-file and full-sequence rotary instrumentation systems. J Endod 2012;38:850-852.

14. Robinson JP, Lumley PJ, Cooper PR, Grover LM, Walmsley AD. Reciprocating root canal technique induces greater debris accumulation than a continuous rotary technique as assesed by 3-dimensional micro-computed tomography. J Endod 2013;39:1067-1070.

15. Poggio C, Dagna A, Chiesa M, Scribante A, Beltrami R, Colombo M. Effects of NiTi rotary and reciprocating instruments on debris and smear layer scores: an SEM evaluation. J Appl Biomater Function Mater 2014:12(3):256-262. 